\title{
Modeling the daily mean values of regional geomagnetic total force field changes in Japan
}

\author{
Satoshi Fujiwara ${ }^{1 *}$, Teruaki Nishiki² ${ }^{2}$ Hiroki Shirai ${ }^{2}$, Hideo Hamazaki $^{2}$, and Vadim P. Golovkov ${ }^{3}$ \\ ${ }^{1}$ Mizusawa Geodetic Observatory, Geographical Survey Institute, 42-2 Uchi-kumagasawa, Kuroishi-machi, Mizusawa, Iwate 023-0101, Japan \\ ${ }^{2}$ Geographical Survey Institute, 1 Kitasato, Tsukuba, Ibaraki 305-0811, Japan \\ ${ }^{3}$ Institute of Terrestrial Magnetism, Ionosphere and Radiowave Propagation (IZMIRAN), Troitsk, Moscow region 142092, Russia
}

(Received May 17, 2000; Revised October 4, 2000; Accepted October 4, 2000)

\begin{abstract}
A spatial-temporal model of the daily mean values of the geomagnetic total force field was constructed for Japan for the time interval from 1997 to 1999 . The daily mean data of six geomagnetic observatories and nine continuous geomagnetic stations in Japan were used for the model. Temporal functions of the model were constructed using the Natural Orthogonal Components method, and the spatial functions are polynomial functions of the horizontal positions. The accuracy of the model is within approximately a few nT, and it can be used for eliminating external fields for geomagnetic surveys or detecting local geomagnetic changes for monitoring crustal activities. Each function, which is calculated using the mathematical (statistical) method, has physical meaning, such as the intensity of the globally symmetrical equatorial ring current and secular changes in the main field. The temporal and spatial functions can be then used for geophysical study of the Earth.
\end{abstract}

\section{Introduction}

Since the geomagnetic field observed on the Earth's surface contains changes due to the Earth's external field and the internal core, common changes are observed in a rather wide region. We need to eliminate the common changes in order to detect small local changes in the geomagnetic field such as piezomagnetic effects in the Earth's crust and volcanomagnetic effects.

The easiest way to eliminate common changes is to take the simple difference between the data at a field station and the data observed simultatiously at a reference station. The simple difference will be reasonable if the two stations are close to each other, but this method cannot sufficiently cancel out common geomagnetic activity in some cases. Rikitake (1966) used weighted differences and Steppe (1979) used a weighted linear combination of several stations for the reference data in order to solve this problem. Kadokura (1990) used the Principal Component Analysis (the Natural Orthogonal Components method) to find the common changes, which are usually the principal part of the geomagnetic changes. Although these improved methods are of higher quality than the simple difference method, we have to determine the parameters using data observed at each station.

We can get the geomagnetic values at any spatial point and at any time in the model without additional analysis for a new station by using spatial-temporal models of the geomagnetic field such as the IGRF (IAGA Division V, Working Group 8,

\footnotetext{
*Now at Research and Development Bureau, Science and Technology Agency, 2-2-1 Kasumigaseki, Chiyoda, Tokyo 100-8966, Japan.

Copy right(c) The Society of Geomagnetism and Earth, Planetary and Space Sciences (SGEPSS); The Seismological Society of Japan; The Volcanological Society of Japan; The Geodetic Society of Japan; The Japanese Society for Planetary Sciences.
}

1995). The global model IGRF has many discrepancies in Japan (Burdelnaya et al., 1999). One in particular is that the time variation model cannot be used for the daily mean. We developed a new regional model which accurately describes the geomagnetic field variations in Japan and can determine time variations of a few weeks. The new model should have functions like the IGRF, which has position (latitude and longitude) and time inputs (day, month and year) and an output for the model value. Contrary to Burdelnaya et al. (1999), this model must use the daily mean time series to expand the data into temporal functions (e.g. Golovkov and Zvereva, 1998).

The present model study has two main purposes. One is to detect local variations of several $\mathrm{nT}$ for reasons including eliminating the external field for geomagnetic survey data at field stations, and the other is to obtain a secular variation field for geophysical study of the Earth. The time interval for the model should be as short as one day for crustal activity and other studies.

\section{Data and Method}

The Geographical Survey Institute (GSI) has been conducting geomagnetic surveys in Japan since 1948. One of the main purposes of the surveys is to detect anomalous changes of the Earth's surface magnetization due to crustal activities such as earthquakes and volcanic activities. Eleven continuous geomagnetic stations with proton and fluxgate magnetometers were installed in 1996 because continuous observation is greatly superior to repeat observations at field stations for this purpose. The data is automatically transmitted to the GSI through public telephone lines. We also use data from 6 GSI (MIZ, ESA and KNZ) and the Japan Meteorological Agency (MMB, KAK and KNY) geomagnetic observatories 
Table 1. Average RMS at 15 stations for each maximum number of NOCs $(k)$.

\begin{tabular}{ccccccccc}
\hline$k$ & 1 & 2 & 3 & 4 & 5 & 6 & 7 & 8 \\
\hline RMS (nT) & 2.27 & 0.53 & 0.41 & 0.30 & 0.25 & 0.21 & 0.18 & 0.15 \\
\hline
\end{tabular}

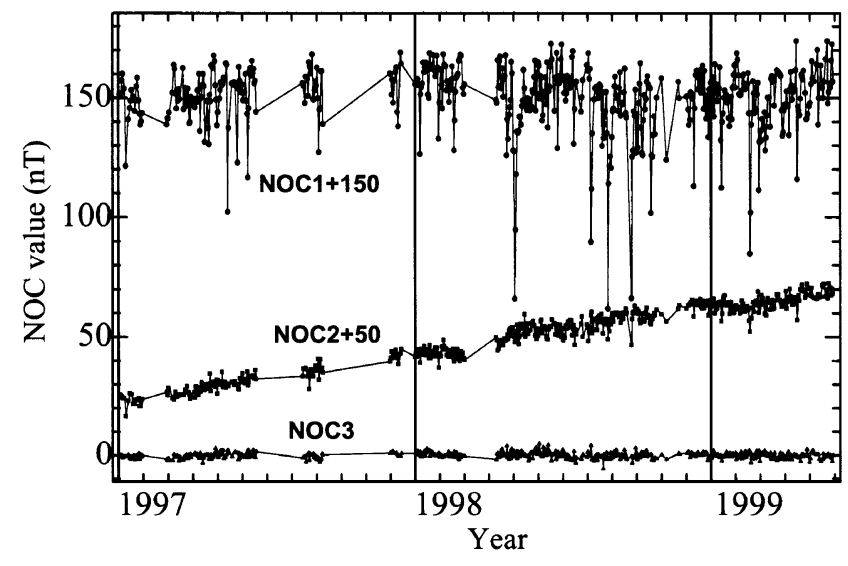

Fig. 1. First three NOCs - temporal functions of the model.

to construct the model. We did not include the data from two continuous stations that contained large artificial noises (traffic and construction near the stations) and used 15 stations in our model. All data is obtained every minute and averaged to get the daily mean.

Burdelnaya et al. (1999) constructed a yearly geomagnetic model over the Far East. We followed their method to develop a geomagnetic field change model for the main area of Japan which covers the two and a half year time interval from January 1997 to June 1999. The analysis is divided into two parts. One is an analysis of the temporal function $(T)$ and the other is an analysis of the spatial function $(X)$.

$$
H(i, \phi, \lambda)=\sum_{k} T_{k i} X_{k}(\phi, \lambda) .
$$

$H$ is a geomagnetic value at geographical coordinates $\phi$ and $\lambda$, and $i$ donates the $i$-th date. We used the Natural Orthogonal Components (NOCs) method for the temporal function analysis. Then we obtained 15 NOCs for each day (Fig. 1). The NOCs have orthogonality with each other, and the power of the NOCs decays as the order $(k)$ decreases. At the same time, residual total variations decay as the number of significant NOCs increases, and local changes in the residual will disappear with too many NOCs. Since the NOCs method minimizes the total amount of variations, which are usually common changes and occasionally large changes at only one station, large artificial noises or large local variations at only one station will significantly affect the model. Therefore, we should determine the total number of NOCs to reduce the common changes and also to keep the local changes. Although we decided to use the first five NOCs according to Table 1, we need a method to find the optimum number of NOCs. Using good observation data is first in importance in practical modeling. Second is a method for automatically detecting large local noises such as a regressive modeling algorithm that will greatly assist visual data inspection. It is also important for good modeling to determine the temporal function in a wider region of interest (Burdelnaya et al., 1999).

In the next step, we calculated the spatial function $X$ for each of the five NOCs using the least square method. We chose the Legandre polynomials on the $(x, y)$ plane as $X$. The second degree is probably sufficient for the maximum degree of the polynomials because the location of the observatories (see Fig. 2) is not uniform. Each of the first five NOCs was spatially interpolated with the second degree Legandre polynomials because the NOCs are only obtained at the observatories used in this study while $X$ needs to be obtained everywhere in the region. Figure 2 shows the spatial function $X$ for the first three NOCs $\left(\mathrm{NOC}_{1}, \mathrm{NOC}_{2}, \mathrm{NOC}_{3}\right)$. This modeling offers us the advantage of being able to determine the parameters without any geophysical knowledge of the temporal and spatial functions because of mathematical (statistical) modeling.

\section{Results}

Figure 3 shows the residual of the daily means of the geomagnetic total force after subtracting the five principal modes. The average RMS is $0.5 \mathrm{nT}$. The residual contains errors from the model and local geomagnetic variations including the effects of ocean currents, thermal-induced changes (Utada et al., 2000), artificial noises, and other factors. Figure 3 generally shows the scatter of short day to day periods and long period changes which are mainly longer than seasonal variations. Since the long period changes do not correlate with each other, they are probably local changes.

Two steps (jumps in the value) found at HAG (in September 1998 and March 2000) are smaller than $1 \mathrm{nT}$ but they are clearly identified. Several noise pulses also found at TTK are probably artificial noises at TTK, but those at ESA and HAR cannot be regarded as noises because they appeared simultaneously on the same days. This is probably an effect of the common geomagnetic field remaining at ESA and HAR. Local inclination anomalies likely created these residuals because the inclination is larger at HAR by $0.5^{\circ}$ and smaller at ESA by $0.5^{\circ}$ than the average directional geomagnetic anomalies (First Geodetic Division, 1993). Even if the disturbing common field is uniform, the resulting fields on the nonparallel local total force field are different from each other (e.g. Davis et al., 1981).

We show an application of the model at field stations for monitoring volcanic activity (Fig. 4, Fujiwara et al., 2000) to check the precision of the model. Mount Iwate, a volcano situated in northern Japan that last erupted in 1919, became active again recently. On September 3, 1998, a large earthquake $(\mathrm{Mw}=6.1$ ) occurred to the southwest of the volcano, and there is likely relationship between the earthquake and the volcanic activity. The data at MAT and SZK in Fig. 4 


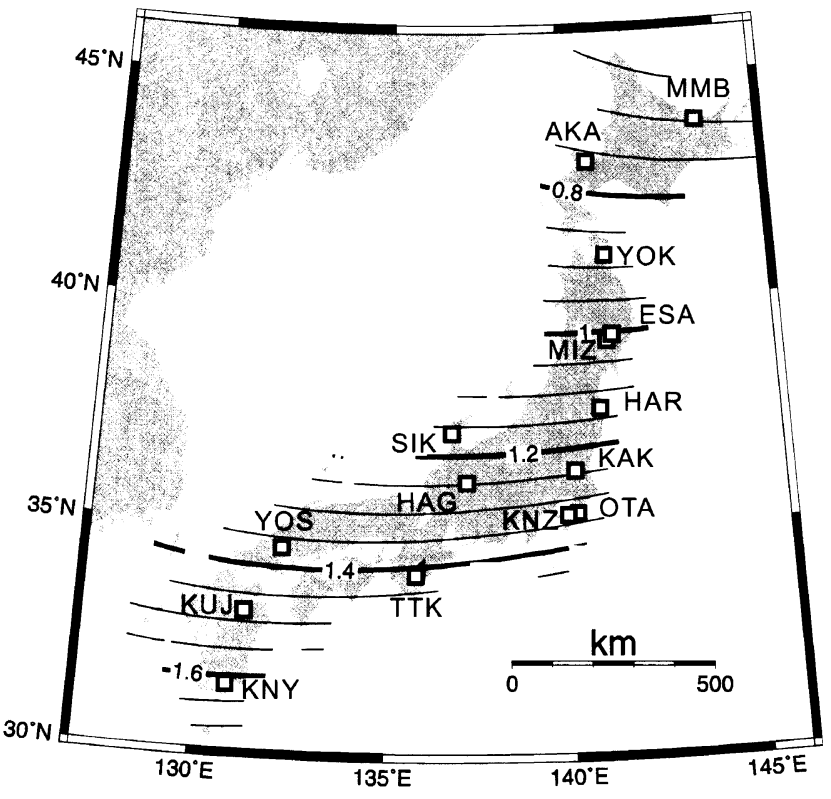

Fig. 2(a). Spatial function for $\mathrm{NOC}_{1}$ (no unit). Location of observatories and continuous stations used in this study is shown as squares.



Fig. 2(b). Spatial function for $\mathrm{NOC}_{2}$ (no unit).

were not used to construct the model. Figure 4 shows the residual of the magnetic daily mean total force after subtracting the model. We could clearly find a decrease of about $1 \mathrm{nT}$ at MAT station from July to September, and the data at SZK station does not show such changes. Since the change at MAT was likely related the volcanic activity, this assures us that the model has sufficient precision of about $1 \mathrm{nT}$.

\section{Discussion and Concluding Remarks}

The temporal function for $\mathrm{NOC}_{1}$ (Fig. 1, upper curve), the largest common change, and the daily mean of Dst (Sugiura

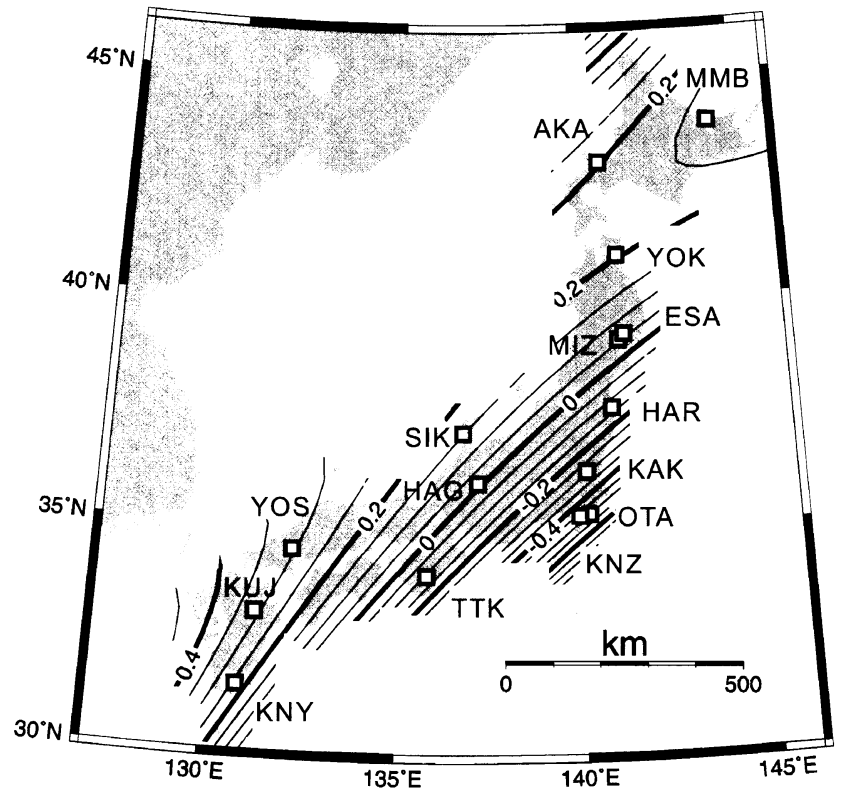

Fig. 2(c). Spatial function for $\mathrm{NOC}_{3}$ (no unit).

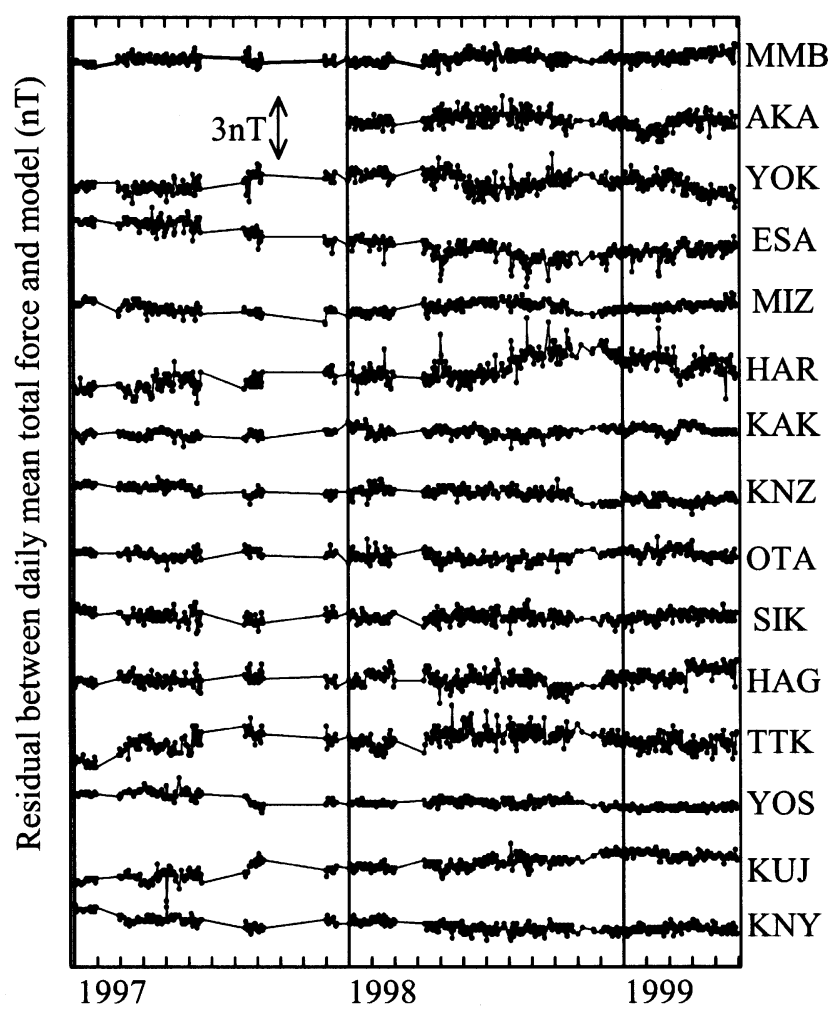

Fig. 3. Residual of daily mean geomagnetic total force after subtracting the model.

and Kamei, 1991; WDC-C2 Kyoto, 2000) closely correlate to each other (Fig. 5). Furthermore, the spatial distribution of $\mathrm{NOC}_{1}$ (Fig. 2(a)) generally shows a latitude function. The $D s t$ index represents the intensity of the globally symmetrical equatorial ring current. The ring current depresses the horizontal geomagnetic component on the surface of the Earth and its intensity is proportional to the cosine of the 


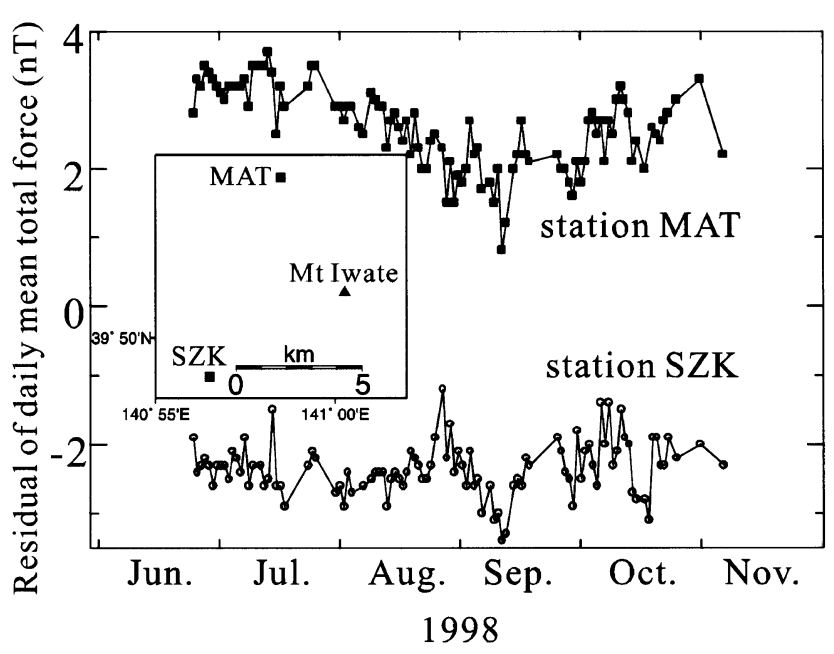

Fig. 4. Residual of daily mean total force after subtracting the model at field stations near Mount Iwate volcano situated in northeastern Japan. The location of the stations and Mount Iwate is shown in the figure.

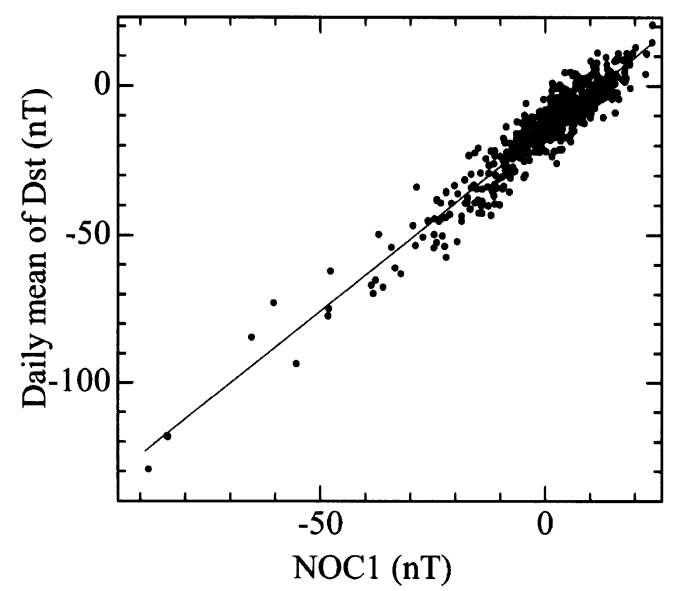

Fig. 5. Relationship between $\mathrm{NOC}_{1}$ and daily mean of Dst (WDC-C2 Kyoto, 2000). Correlation coefficient is 0.95 .

geomagnetic latitude. Although the ratio of the cosine of the geomagnetic latitude from the southern part to the northern part of Fig. 2(a) is about 1.2, the $\mathrm{NOC}_{1}$ ratio (Fig. 2(a)) is more than 2 . This is mainly because the effect of the Dst on the total intensity is more complicated than it is on the horizontal component, and the inclination in the northern part is larger by $15^{\circ}$ than that in the southern part (First Geodetic Division, 1993). The effect of the declination distribution is smaller than that of the inclination, however, because the spatial variation of the declination is a maximum of $4^{\circ}$ in Fig. 2.

Figure 1 suggests that $\mathrm{NOC}_{2}$ is a secular change of the geomagnetic field. Although the secular change was quite linear and stable in the time period for this study, the secular changes had been unstable and changed randomly in the past (Tazima et al., 1976). Moreover, the spatial variation of the secular changes had changed with time. Consequently, long period secular changes will be complicated and probably cannot be described with only one NOC, and the spatial function for $\mathrm{NOC}_{2}$ (Fig. 2(b)) will change.

Although $\mathrm{NOC}_{3}$ is much smaller than $\mathrm{NOC}_{1}$ and $\mathrm{NOC}_{2}$ (Fig. 1), the spatial function for $\mathrm{NOC}_{3}$ (Fig. 2(c)) shows more complicated geographical patterns. Since this spatial distribution is similar to that of the induction arrow in this area (Fujiwara and Toh, 1996), the local electromagnetic induction field related to the local electric conductivity structure is likely to be the cause of the component. This is because the relative ratio and direction of the induced internal field to the external field is not stable due to the external vertical component contamination change (e.g. Fujiwara and Toh, 1996).

In conclusion, the two main sources of the common changes in the daily mean geomagnetic total force in Japan are the following;

(1) The intensity of the globally symmetrical equatorial ring current (external origin) and its combination with the inclination distribution (local internal magnetic anomaly) and

(2) Secular changes of the main field (internal origin).

There is also a possible source of electromagnetic induction in the Earth associated with external geomagnetic activity. Since the sources are clearly separated in our model, we can use the temporal functions for geophysical research. This modeling method is purely based on the statistical features of the data without any constraints obtained from a priori knowledge of the underlying physical processes, but the output of the temporal and spatial functions can be used for physical modeling. Conversely, if we have a priori physical data (Dst, magnetic anomalies and local induction), hybrid statistical modeling using the physical data can improve the precision of the model.

Acknowledgments. The authors wish to thank the members of the GSI for providing geomagnetic data, and the Japan Meteorological Agency for providing MMB, KAK and KNY data. We thank $\mathrm{H}$. Utada and I. Fujii for their careful reviews and constructive comments.

\section{References}

Burdelnaya, I. A., S. V. Filippov, V. P. Golovkov, S. Fujiwara, T. Tanabe, S. Nishi, M. Kaidzu, and S. Matsuzaka, Regional orthogonal models of the geomagnetic field changes over the Far East, Earth Planets Space, 51, 287-296, 1999.

Davis, P. M., D. D. Jackson, C. A. Searles, and R. L. McPherron, Detection of tectonomagnetic events using multichannel predictive filtering, $J$. Geophys. Res., 86, 1731-1737, 1981.

First Geodetic Division, Magnetic charts for the epoch 1990.0, Bull. Geograph. Survey Inst., 38, 1-3 (with charts), 1993.

Fujiwara, S. and H. Toh, Geomagnetic transfer functions in Japan obtained by first order geomagnetic survey, J. Geomag. Geoelectr., 48, 1071-1101, 1996.

Fujiwara, S., H. Hamazaki, and T. Kadowaki, Continuous observation of geomagnetic total field to monitor the activity of Mount Iwate volcano, J. Geograph. Survey Inst., 93, 12-18, 2000 (in Japanese).

Golovkov, V. P. and T. I. Zvereva, Expansion of geomagnetic variations inside of the year into natural orthogonal components, Geomag. Aeronomy, 38, 140-145, 1998 (in Russian).

IAGA Division V, Working Group 8, International geomagnetic reference field, 1995 revision, J. Geomag. Geoelectr., 47, 1257-1261, 1995.

Kadokura, S., A new method for data analysis of precise observation of geomagnetic secular variation, Proc. Conductivity Anomaly Res. 1990, 151-158, 1990 (in Japanese).

Rikitake, T., Elimination of non local changes from total intensity values 
of the geomagnetic field, Bull. Earthq. Res. Inst., Univ. Tokyo, 44, 10411070, 1966.

Steppe, J. A., Reducing noise in tectonomagnetic experiments by linear regression, J. Geophys. Res., 84, 3063-3067, 1979.

Sugiura, M. and T. Kamei, Equatorial Dst Index 1957-1986, IAGA Bulletin No. 40, 246 pp., ISGI Publ. Office, France, 1991.

Tazima, M., H. Mizuno, and M. Tanaka, Geomagnetic secular change anomaly in Japan, J. Geomag. Geoelectr., 28, 69-84, 1976.

Utada, H., M. Neki, and T. Kagiyama, A study of annual variations in the geomagnetic total intensity with special attention to detecting volcanomagnetic signals, Earth Planets Space, 52, 91-103, 2000.

WDC-C2 Kyoto, Dst index service, http: //swdcdb.kugi.kyoto-u. ac.jp/dstdir/index.html, 2000.

S. Fujiwara (e-mail: fujiwara@gsi.go.jp), T. Nishiki, H. Shirai, H. Hamazaki, and V. P. Golovkov 